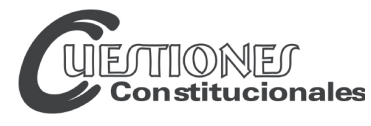

Revista Mexicana de Derecho Constitucional

Núm. 26, junio-diciembre 2012

\title{
LA DINÁMICA CONSTITUCIONAL: UN SISTEMA CAÓTICO
}

\author{
CONSTITUTIONAL DYNAMICS: A CHAOTIC SYSTEM
}

\author{
Rosa María DE LA TORRE TORRES* \\ Salvador JARA GUERRERO**
}

\begin{abstract}
RESUMEN: El presente trabajo, es resultado de un ejercicio de reflexión desde dos disciplinas que en apariencia están desvinculadas: el derecho y la física.

En el texto del artículo, los autores exploran, desde una versión interdisciplinaria, la idea de concebir el sistema constitucional más que como un sistema completo y autopoyético, como un sistema dinámico, complejo e incluso caótico.

Partiendo del estudio de la teoría de los sistemas complejos establecida en la Física, se incorporan elementos interpretativos y argumentativos para identificar la dinámica constitucional como un sistema no lineal, evidentemente dinámico y con una complejidad cercana al caos.

Pero es de resaltar que uno de los objetivos principales de los autores es, eliminar toda carga peyorativa al adjetivo caótico, abriendo la posibilidad a una concepción favorable del caos en el sistema constitucional.
\end{abstract}

Palabras clave: teoría de sistemas, sistema constitucional, sistemas complejos, sistemas dinámicos.
ABSTRACT: This paper is product of an exercise of reflection and communication between two seemingly opposing disciplines: Law and Physics. In this text the authors explore, from an interdisciplinary approach, the idea of conceiving a constitutional system beyond a complete and autopoietic system, and much more like a dynamical, complex and even chaotic system. Concepts taken from the theory of dynamical or complex systems established in Physics are used as motivation to interpret and identify the dynamics of a constitutional system as nonlinear and as one that bears a complexity nearly chaotic. However, it must be said that one of the main aims of the paper is to get rid of all negative views that are usually associated with chaos. The purpose is to show a favorable conception of chaos within a constitutional system.

Descriptors: theory of systems, constitutional system, complex systems, dynamical systems.

* Doctora en derecho constitucional; investigadora del Centro de Investigaciones Jurídicas de la Facultad de Derecho y Ciencias Sociales de la Universidad Michoacana de San Nicolás de Hidalgo.

** Doctor en filosofía; investigador de la Facultad de Ciencias Físico-Matemáticas de la Universidad Michoacana de San Nicolás de Hidalgo. 


\section{INTRODUCCIÓN}

La pregunta por lo bueno, la belleza, el bien, el buen gobierno, la buena ley o la justicia nos remite irremediablemente al problema de los universales y a la tensión entre lo general y lo particular. Y posiblemente esta problemática no haya sido mejor ilustrada que por Voltaire y su magnífico humor. En Cándido, el personaje de Cacambo representa esa sabiduría particular, práctica y popular que se obtiene con la experiencia de la vida, frente a la actitud metafísica del doctor Panglos, filósofo optimista, pensador teórico, teólogo-cosmo-nigólogo. El sabio doctor defiende con convicción y vehemencia que éste es el mejor de los mundos posibles y que a fin de cuentas las cosas son como son porque así deben ser, puesto que ya son lo mejor posible, está es su finalidad. El doctor Panglos sólo habla, en situaciones difíciles, habla, frente a las catástrofes, habla. Sólo habla y no es capaz de ver los defectos o las impurezas de nada, no reconoce ninguna tragedia, y no sólo las niega sino que las justifica, son así porque así deben ser.

Inspirada en Panglos, Katya Mandoki ha denominado como el síndrome panglosiano ${ }^{1}$ a una actitud que no nos permite ver los defectos, las imperfecciones de las cosas, sino sólo lo bueno y lo hermoso, de tal manera que nos convence de que lo que tenemos es siempre lo mejor posible. Así, con ese optimismo, diríamos que la noción de la belleza, la verdad, la buena ley o la justicia no son construcciones que dependen de las características propias de los grupos sociales y culturales sino hechos ontológicos, nociones que existen per se y que pueden ser alcanzadas independientemente de las convenciones sociales y culturales o de las particularidades de cada caso. El síndrome de Panglos fabrica ídolos, mitos y fetiches de las cosas al no reconocer sus imperfecciones e incluso justificarlas con el argumento de que "ya son lo mejor posibles".

La contraparte de Panglos, Cacambo, muestra una virtud prudencial basada en la experiencia que probablemente esté inspirada en el concepto de saber popular o el buen sentido común que Aristóteles identificara como la Frónesis; que es una suerte de sabiduría práctica y prudente que no se opone a la pretensión de las verdades universales, representadas por la Sofía, sino que más bien se coloca como su conciencia, como el contrapeso necesario de una reflexión razonable — más que sólo racional—y prudente

1 Mandoki, Katya, Estética cotidiana y juegos de la cultura, México, ConacultaFONCA, 2008, p. 51. 
de las pretensiones de verdad absoluta y de universalidad, con base en las particularidades de cada caso. Esta virtud sólo puede adquirirse a través de la experiencia práctica cotidiana.

La actitud del doctor Panglos es una sátira dedicada al optimismo del pensamiento racionalista ilustrado, y es una burla al sueño cartesiano de lograr un conocimiento claro, distinto y universal a través de la razón. A fin de cuentas se trata de una crítica al pensamiento moderno, que ha permeado todas las disciplinas y saberes.

El derecho ha sido, desde sus más remotos orígenes, receptáculo de los influjos del pensamiento humano; la evolución de la doctrina, la filosofía y la epistemología jurídicas reflejan los devenires de las concepciones filosóficas sobre el hombre como individuo, como parte de una sociedad o Estado, las relaciones del ser humano con la divinidad, con el poder y con sus pares.

La evolución del hombre puede medirse con respecto al desarrollo de sus instituciones. El derecho, sea tal vez, uno de los productos culturales que cristaliza con mayor precisión las tendencias del pensamiento humano, la disciplina jurídica construye ideales y eleva paradigmas.

Uno de los paradigmas elevados desde la antigüedad es pensar el derecho no como un mero conjunto de normas jurídicas rígidas, como un dogma o fetiche, o bien como un simple catálogo, sino como un sistema complejo donde coexisten diversos valores, principios y normas, sean de carácter religioso, moral o jurídico en sentido estricto. ${ }^{2}$ El derecho se ha concebido como un sistema donde los equilibrios, pesos y contrapesos entre las normas jurídicas emanadas del quehacer humano deben atender a principios o valores múltiples, tanto metafísicos como los derivados de la experiencia.

El objetivo de este trabajo es revisar la concepción del derecho como sistema y algunas de las teorías que sobre los sistemas jurídicos se han pensado; para ello, se recurrirá a una referencia sobre los sistemas desde una perspectiva transdisciplinaria para comprender, en primer lugar la teoría de los sistemas y su aplicación jurídica y en segundo lugar, proponer una reconcepción del derecho como un sistema no solamente abierto, sino

2 En este sentido recomendamos el texto de Alejandro Nieto Crítica de la Razón Jurídica, Madrid, Trotta, 2007. Recomendamos especialmente, para dar fundamento a estas afirmaciones, la lectura del capítulo 3 “Ámbito: lo jurídico y lo no jurídico”, pp. 65 y ss. 
como un sistema dinámico y, aventurándonos un poco más, como un sistema caótico, lo que lejos de demeritarlo, hace justicia a su complejidad.

Finalmente, queremos destacar que este trabajo es un reflejo de la fructífera comunicación que puede darse entre las ciencias naturales, sociales y las humanidades, incorporando aportaciones teóricas y conceptuales de una ciencia para explicar los fenómenos observables desde otras disciplinas.

\section{SISTEMAS CERRADOS Y SISTEMAS DINÁMICOS. \\ APORTES PRELIMINARES}

Si bien una de las pretensiones de la modernidad, a través de la ciencia, fue la de abandonar los prejuicios medievales, también es cierto que esto permitió el nacimiento de otros ídolos, la ilusión de una objetividad radical, la seguridad de una interpretación única de la realidad y la confianza optimista de que en todos los casos es posible encontrar o des-cubrir la interpretación verdadera, la respuesta correcta. ${ }^{3}$

Dos supuestos permitieron este movimiento del pensamiento medieval a la racionalidad científica, especialmente en la Física. En primer lugar la convicción de que la naturaleza no cambia de manera fundamental, que los fenómenos y los objetos naturales son invariantes en esencia, lo que cambia es accidental y poco importante. Esta permanencia esencial de los fenómenos permite el estudio de éstos de manera reproducible y controlada y, por tanto, pueden estudiarse como sistemas cerrados o aislados de perturbaciones o efectos externos. De esta forma en cada fenómeno las variables importantes que intervienen son pocas y conocidas, por lo que pueden ser manipuladas, mientras que se supone que el resto tienen poca influencia y son sencillamente ignoradas.

El segundo supuesto es el de la doctrina de la causalidad. ${ }^{4}$ En ésta se da por hecho que la misma situación produce siempre las mismas consecuencias, o dicho de otra manera que la misma causa produce siempre y exactamente los mismos efectos. En muchos casos esto es cierto, en los

3 En este sentido vale mucho la pena recurrir al texto de María Luisa Balaguer "La interpretación de las normas y la posibilidad de una sola respuesta correcta", en Morodo, Raúl y Vega, Pedro de (coords), Estudios de Teoría del Estado y derecho constitucional en honor de Pablo Lucas Verdú, Tomo I, Madrid, Dykinson-UCM, 2000.

4 D'Abro, A., The Rise of the New Physics, Nueva York, Dover Publications, 1951, pp. 45 y 46. 
fenómenos muy simples, pero en la mayoría de nuestras experiencias, si pudiéramos viajar al pasado y vivir nuevamente, lo más seguro es que, como en la película de Corre Lola corre, cualquier pequeñísima modificación produciría cambios enormes. Así que este supuesto de causalidad aplica solamente a los fenómenos que son absolutamente reproducibles, los que es posible reproducir con absoluta precisión a partir de las condiciones en que se originan, denominadas condiciones iniciales. Para ello es necesario repetir exactamente el inicio del proceso, controlar las variables que le afectan y evitar interferencias externas.

Las dos suposiciones anteriores aplican para fenómenos físicos relativamente sencillos, ordenados y regulares que pueden ser descritos por medio de leyes matemáticas. Leyes que no sólo los explican sino que además permiten reconstruir su pasado y predecir con singular precisión su futuro. Lo extraordinario de una ley física es que determina a priori la evolución o el desarrollo de un sistema, permite que conozcamos su futuro, y basta para ello con conocer el estado de cosas inicial con precisión.

Pero hay que enfatizar que en los sistemas cerrados ideales un pequeño cambio o error en las condiciones iniciales produce un error igualmente pequeño en las predicciones o efectos futuros. Con el tiempo la realidad nos ha mostrado que el mundo es mucho más complejo, que las dos suposiciones descritas se cumplen en condiciones muy especiales, que los sistemas cerrados son más bien escasos, ${ }^{5}$ que la mayoría de los fenómenos se desarrollan en sistemas abiertos y que un pequeño cambio en las condiciones iniciales puede producir efectos insospechados.

El estudio de los fenómenos en sistemas cerrados, aunque supone una simplicidad excesiva, fue lo que permitió a la ciencia mantener válidamente los principios de causalidad, predictibilidad y universalidad, pero a la vez que esos mismos principios posibilitaron un avance sin precedentes en el saber y fueron condiciones para su éxito, también constituyeron los límites

5 Es importante señalar que aún en los sistemas considerados cerrados pueden darse variaciones al mismo sistema que ponen en evidencia que al final, todos son sistemas abiertos; como un ejemplo podemos pensar en 1 movimiento de la Luna alrededor de la tierra el cual podemos predecir suponiendo que la única fuerza que actúa sobre el satélite es la gravedad de la Tierra, si así fuera tendríamos un sistema perfectamente cerrado, conformado por la Tierra y la Luna. Sin embargo, el movimiento lunar se ve afectado así sea de manera aparentemente poco importante por la atracción de otros planetas y por el Sol, lo que en mediciones largas de tiempo puede provocar cambios drásticos en su movimiento. 
inmanentes de la actividad científica moderna al convertir la metodología científica y sus productos en una especie de dogma, y por tanto, en un obstáculo para el cambio. ${ }^{6}$

Desgraciadamente, en la realidad no existen los sistemas cerrados, lo que las ciencias naturales han hecho es construir modelos simples que puedan ser estudiados y que permiten predecir razonablemente los fenómenos bajo estudio. Cuando ha sido posible aislar suficientemente un fenómeno y controlar adecuadamente sus variables ha sido también posible estudiarlo como si prácticamente no interactuara con su exterior, es decir, estudiarlo como un sistema cerrado idealizado. Quizá sólo el universo entero, considerado como un todo, sea el único sistema verdaderamente cerrado. Resumiendo, podemos decir que un sistema cerrado es un sistema rígido porque sus elementos, aunque interactúan, siempre son los mismos, en cambio un sistema abierto se alimenta continuamente del exterior y, por tanto, cambia.

Aún en la física y otras ciencias naturales, cuando el estudio de los fenómenos pasó a detalles más finos, la presencia de variables del entorno resultaron ser muy importantes. Pero el descubrimiento más interesante fue que aun cuando se conozcan las variables y se pueda tener un control más o menos fino de ellas, en algunos sistemas (más de los que se hubiera pensado y deseado) un pequeño cambio produce cambios enormes en los efectos, lo que obligó a abandonar la suposición de que en los sistemas deterministas un pequeño error en las condiciones iniciales se refleja en una pequeña variación en los resultados. ${ }^{7}$

Estos sistemas abiertos denominados caóticos muestran aparente azar, accidentalidad absoluta o comportamiento impredecible, a pesar de estar gobernados por leyes deterministas. Si bien las leyes que explican estos fenómenos o a las que "obedecen" se cumplen estrictamente, siguen resultando impredecibles en virtud de las variaciones en las condiciones iniciales, así sean estas muy pequeñas. Uno de los mejores ejemplos de estos sistemas es el clima: las leyes que lo rigen son conocidas, y sin embargo su predicción es imposible más allá de algunos días. La gran sorpresa fue que estos

6 Kuhn, Thomas, La función del dogma en la Investigación Cientifica, Valencia, Teorema, 1979, p. 5.

7 Vale la pena resaltar que tal vez el ejemplo más accesible de un sistema abierto y complejo sea el de un individuo. Un cambio en el estado de ánimo puede dar lugar a cambios físicos y a la inversa, y no siempre los grandes cambios en el exterior producen cambios significativos. 
sistemas pueden ser relativamente simples, y que de hecho muchos de los fenómenos conocidos pueden mostrar características caóticas.

Desde el descubrimiento de estos sistemas, y de su abundancia, nuestra visión del mundo ha cambiado, está cambiando, los sistemas simples pueden dar lugar comportamientos complejos y los sistemas complejos pueden dar lugar a comportamientos simples. ${ }^{8}$

En los denominados sistemas complejos o caóticos, aunque las leyes que los rijan sean sencillas y conocidas, tienen un número muy grande de componentes de naturaleza muy distinta, en sus procesos reina la no linealidad: un parámetro puede estar afectado por múltiples causas. En los sistemas complejos: una misma causa puede provocar multitud de efectos. ${ }^{9}$

La suposición de que la regularidad y lo causal determinista eran la norma y lo contingente un accidente llevó a considerar el mundo como si se tratara de un sistema lineal, que podía estudiarse en pedazos. Se estudia independientemente cada trozo y luego simplemente se juntan. Pero en los sistemas no lineales la suma de las propiedades de cada parte no es igual a las propiedades del todo.

Las interacciones entre las partes de un sistema no lineal dan lugar a propiedades emergentes, nuevas, que no pueden explicarse a partir de las propiedades de las componentes. Un ejemplo es un hormiguero donde la colonia de hormigas es capaz de desarrollar tareas sociales, es decir, tareas que sólo son posibles de desarrollar en comunidad pero que cada hormiga por sí sola no realizaría en parte proporcional. El comportamiento social emergente es característico de un sistema complejo y no se puede reducir a las propiedades de cada individuo.

El Premio Nobel Ilya Prigogine ha hecho énfasis en que en esos casos, con frecuencia aparecen elementos azarosos, como si no hubiera leyes, lo que muestra que incluso la realidad física es mucho más que la suma de las partes estudiadas por separado; que existe una diferencia cualitativa entre las partes y el todo. Reconocer esa complejidad y efectuar una relectura dentro de este nuevo contexto de relaciones cambiantes del hombre con la 304.

Gleick, J., Chaos: Making a New Science, Nueva York, Penguin Books, 1987, p.

Cereijido, M., "Del caos de los demonios al caos de los biólogos", Revista Universidad de México, núm. 540, enero de 1996, p. 6. 
naturaleza y de ésta con el hombre es, dice Prigogine, uno de los problemas cruciales de nuestra época. ${ }^{10}$

Las ciencias sociales y en particular el derecho no estuvieron exentas de la influencia cientificista y adoptaron modelos semejantes a los de las ciencias naturales. Pero mientras que algunos fenómenos físicos se pueden reproducir fielmente - en condiciones artificiales y controladas - y entonces las teorías pueden verificarse o falsarse experimentalmente. En cambio, en la historia, el derecho y otras disciplinas, cada evento es único e imposible de repetir. En muchos casos el conocimiento que podemos obtener sobre toda nuestra experiencia pasada es como bien ha dicho Arundhati Roy, como asomarse de noche a una casa vieja por la ventana; vemos cuadros sobre la pared y libros, nuestros ancestros están dentro, pero no podemos entrar y lo que observamos son sólo sombras y lo que escuchamos sólo son susurros. ${ }^{11}$

\section{SISTEMAS JURÍDICOS CERRADOS Y SISTEMAS JURÍDICOS ABIERTOS}

La concepción del derecho como un sistema, como un complejo de partes dinámicamente relacionadas, no es una idea nueva. Desde los primeros ejercicios de reflexión jurídica se entendió que el derecho debe ser comprendido como un conjunto de elementos sistematizados, de una o de otra manera, más o menos positivados, que regulan ciertas relaciones entre individuos y entre los mismos individuos y la autoridad.

Desde esta perspectiva encontraremos dos posturas paradigmáticas en la concepción del derecho como sistema normativo: los que consideran al derecho como un sistema normativo cerrado, y por otro lado, los que lo consideran abierto.

Por ejemplo René David ${ }^{12}$ hace una clara diferenciación entre sistemas jurídicos cerrados y sistemas jurídicos abiertos. Los primeros corresponden a los derechos emanados de la familia romano-germana, de suerte que cualquier caso, al menos en términos teóricos, puede resolverse mediante la interpretación de una regla jurídica preexistente, es decir, de una norma que forma parte del mismo sistema.

${ }^{10}$ Prigogine, Ilya, ¿Tan sólo una ilusión?, Barcelona, Tusquets Editores, 1983, p. 45.

${ }^{11}$ Roy, Arundhati, The God of Small Things, Nueva York, Random House, 1997.

12 David, René, Los grandes sistemas de derecho contemporáneo, 6a. ed., París, Dalloz, 1974, p. 363. 
En cambio, el derecho inglés es un sistema abierto que supone un método para resolver cualquier cuestión de tal modo que no implica reglas de fondo aplicables. Así, la técnica del derecho inglés no es interpretadora de reglas jurídicas, de legal rules, ya que desde la perspectiva del jurista inglés, a un caso nuevo debe corresponder una regla nueva. Es decir, se apela a las características particulares del contexto, se trata de tomar en consideración cualquier factor externo al sistema.

En los sistemas jurisdiccionales anglosajones la función del juez es hacer justicia y no le compete, formular, en términos generales, las reglas que por su alcance, rebasan el litigio en cuestión. En resumen, la concepción inglesa de la legal rule se explica históricamente, el common law es obra de los jueces y está íntimamente ligado a la técnica de las distinciones frente a la interpretación.

La doctrina ha introducido diversas expresiones que cuadran con la apertura de los sistemas jurídicos, esto se refleja principalmente en la apertura de la Constitución. Así en la literatura anglosajona encontramos el término living constitution, acogida por los autores alemanes como lebende Verfassung. En Portugal, el tratadista Gomes Canotilho utiliza el término Constitución adecuada ${ }^{13}$ la cual se entiende adecuada a dimensiones no estrictamente jurídico-normativas.

Aún más ilustrativo es el concepto de Constitución de Von Hayek ${ }^{14}$ cuando habla de una Constitución ideal. El prestigioso premio Nobel antepone como lema a su argumentación el siguiente texto: "En materia jurídica-constitucional y de gobierno conviene siempre conocer lo más perfecto para poder ir aproximando a ese ideal las instituciones existentes, introduciendo, sin embargo, en todo momento, cualquier innovación, de manera tan paulatina que se no cause excesivo trastorno a la sociedad".

La afirmación anterior, nos permite reconocer la extrema sensibilidad, que en la práctica, muestran los sistemas jurídicos a cualquier variación o innovación.

En este trabajo se pretende señalar al derecho como orden normativo abierto, complejo, dinámico e, incluso, caótico y en ese sentido, igual que

${ }_{13}$ Gomes Canotilho, J. J., Dereito constitucional, Coimbra, Almedina, 1993, pp. 197 y ss.

${ }^{14}$ Von Hayek, Friedich, Derecho, legislación y libertad, Madrid, Unión Editorial, 1982, vol. III. pp. 185 y ss. 
como lo señala don Pablo Lucas Verdú, ${ }^{15}$ estamos conscientes que esto entraña un juicio de valor favorable al tema de la apertura y la dinámica del sistema jurídico. En este mismo sentido podemos señalar el término sociedad abierta en oposición a la sociedad cerrada. ${ }^{16}$

Por lo anterior, podemos llegar a intuir una contraposición entre apertura, como concepto positivo y, clausura como algo negativo.

El concepto de apertura se puede encuadrar en el contexto de las sociedades democráticas, el cierre se ubica en el ámbito de las sociedades totalitarias. La frase de Musolinni: "Nada contra el Estado nada fuera del Estado, todo dentro del Estado" resulta ilustrativa a este respecto.

\section{La Constitución como sistema normativo abierto}

En la obra de Karl Popper ${ }^{17}$ sobre la sociedad abierta y sus enemigos encontramos una idéntica idea de bondad respecto a la apertura social. En el pensamiento popperiano, la apertura constitucional, como la sociedad abierta, en cuanto se consideran manifestaciones de un espíritu democrático, implican la relación de estas dos con la publicidad, el pluralismo, la alternancia en el poder y la tolerancia, valores que resultan fundamentales en una democracia.

En un sentido muy similar, el doctrinario brasileño Willis Santiago Guerra Filho, ${ }^{18}$ a la vista de la reforma de la Constitución de 1988, considera que una Constitución ideal es aquélla que contiene un elenco mínimo de principios organizadores del Estado que vinculen su acción en pro de la mejora de las condiciones vitales en nombre de la sociedad sin perderse en reglamentaciones minuciosas y casuísticas que no corregirán los problemas actuales. Así, pues, la Constitución ideal puede ajustarse a la Constitución actual y real de la sociedad o a la de un futuro probable.

En definitiva, el profesor brasileño antes citado, aboga por una apertura y conexión con la realidad subyacente.

15 Lucas Verdú, Pablo, La Constitución abierta y sus enemigos, op. cit., p. 27.

16 En este sentido es indispensable citar a Peter Häberle en su obra Teoría de la Constitución como ciencia de la cultura, Madrid, Tecnos, 2000, 161 pp.

17 Popper Karl, La sociedad abierta y sus enemigos, 4a. reimp. (trad. de Eduardo Loedel), Buenos Aires, Editorial Paidós, 1991, 220 p.

18 Guerra Filho, W. Santiago, Ensayos de teoría constitucional, Fortaleza-Ceara, 1989, pp. 96 у 97. 
La Constitución británica ha sido muy elogiada como paradigma de perfección política ${ }^{19}$ y lo mismo ha sucedido con la Constitución norteamericana. Las dos son para la teoría de la Constitución, desde lo dicho por el vizconde Bryce, modelos flexible y rígido respectivamente, que configuran la convivencia política. Analizando lo anterior podemos concluir, que la rigidez de la Constitución de 1787 no es óbice para su elasticidad, o sea, para acomodarse a las variadas, y a veces, drásticas vicisitudes que le ha tocado vivir al pueblo norteamericano. Es gracias a esa elasticidad y el espíritu cívico de sus dirigentes, y del mismo pueblo, que este Texto Fundamental ha podido alcanzar sus más de dos siglos de vigencia.

Para los norteamericanos su Constitución viene a ser una especie de texto fundamental y sagrado que merece veneración. Leviston recoge una afirmación significativa de Kristol cuando dice que la bandera, la Declaración de Independencia y la Constitución, son la santa trinidad de lo que Tocqueville llamaba la religión civil norteamericana. Alexander Bickel señala que en esta religión secular de la república norteamericana encontramos nuestras visiones del bien y del mal. ${ }^{20}$

Ackerman $^{21}$ subraya, en tiempos más recientes, las virtualidades de la Constitución de 1787. Para descubrir la Constitución hemos de actuar convencidos de que ni Aristóteles, Cicerón, Montesquieu, ni Locke, ni Harrington, ni Kant o Webber nos suministrarán la llave precisa. Los norteamericanos aprendieron mucho de estos autores, sin embargo, edificaron un genuino y peculiar modelo de pensamiento y práctica constitucionales. Una vez que se ha reconstruido ese modelo, se percataron que puede compararse con las reflexiones más profundas sobre la naturaleza de la política de los griegos, de los romanos o de los ingleses.

Para los norteamericanos, describir su Constitución es como describir una parte de ellos mismos. Cuentan con la naturaleza e historia de su propio pasado constitucional y al mismo tiempo configuran el significado que preparan para el presente y el futuro de su país. ${ }^{22}$

19 Lucas Verdú, Pablo, “Alabanza y menosprecio de la Constitución inglesa”, Revista de la Facultad de Derecho de la Universidad de Oviedo, núm. 28, 1956, pp. 315 y ss.

${ }^{20}$ Cfr. lo que dice Stanfrod Levinson en Constitutional Faith, capítulo I: The Constitution in American civil religión, pp. 99 y ss.

${ }^{21}$ Ackerman, Bruce, We the people. 1. Foundations, Cambridge, Harvard University Press, 1991, p. 3.

22 Idem. 
El racionalismo crítico, compartido por Popper en el campo sociológico y en el ámbito constitucional por Peter Häberle, ofrece una perspectiva epistemológica y metodológica para poder encarar el problema. Ahora bien, como no es conveniente olvidar las facetas estimativas del asunto de estas se aclaran añadiendo el enfoque del iusnaturalismo crítico. Es decir, el derecho como sistema abierto y dinámico entraña la apertura al hombre, a su dignidad, a sus derechos y al libre desarrollo de su personalidad, si bien como escribe Miranda ${ }^{23}$ el concepto de Constitución se ha convertido hoy en un concepto neutro, abierto a contenidos políticos, económicos y sociales divergentes que se traducen en tipos constitucionales característicos.

La Constitución en cuanto tal es la sede de los valores jurídicos básicos recogidos en la comunidad política. Es expresión inmediata de la idea de derecho en ella triunfante. Es el estatuto del poder que se pretende al servicio de esa idea y el cuadro de referencia de la sociedad a ese poder. ${ }^{24}$

Si bien, el concepto de Constitución abierta, entendida como una Constitución abierta a elementos jurídicos supra normativos, como los que devienen de la fuente internacional o supranacional, es un concepto cada vez más aceptado, hay dos conceptos sobre la apertura constitucional que aún suscitan grandes debates y profundas reflexiones: la apertura hacia las costumbres y expresiones axiológicas minoritarias - generadas, en gran medida, por los localismos y regionalismos culturales y étnicos- y la apertura hacia la construcción jurisdiccional.

En el primero de estos supuestos implica que la Constitución no solamente es el receptáculo de unos valores universalmente aceptados, sino también es recipiente de valores que devienen de culturales locales, de expresiones no mayoritarias; esto implica necesariamente la convivencia, dentro del texto constitucional de una pluralidad de tendencias y de cuerpos axiológicos. Sin embargo, esta convivencia debe darse en sintonía, permitiendo el desarrollo armónico de todas las expresiones culturales, sociales, religiosas y étnicas que se reflejan en el texto constitucional como valores implícitos en derechos específicos.

Estos valores se convierten en atractores de un sistema dinámico y caótico como es el derecho y será el juez constitucional quien tengan la imprescindible labor de identificarlos, y esto nos lleva necesariamente a

23 Miranda, Jorge, Manual de direito constitucional, t. IV, Direitos fundamentais, 2a. ed. Coimbra, 1993.

24 Idem. 
un tercer enfoque sobre el concepto de apertura: la Constitución entendida como un sistema abierto a la construcción jurisdiccional.

Reconocemos que este sea tal vez el aspecto más debatido aún sobre la apertura del sistema constitucional. Entender que el derecho no es, sino que se construye es abrir la puerta a un campo fértil para el debate iusfilosófico y es precisamente este uno de los objetivos del presente trabajo.

\section{LA CONSTITUCIÓN COMO SISTEMA DINÁMICO}

Como hemos venido esbozando a lo largo de este trabajo, el derecho, en el siglo XVIII, invadido por el ímpetu racionalista y positivista, construyó algunos dogmas, siguiendo los ideales de la modernidad, y esto se refleja especialmente en las cuestiones constitucionales. Ideas como el derecho concebido como un sistema cerrado, perfecto, completo y autopoyético sirvieron de base para la construcción de un concepto de Constitución como un fiel reflejo de la "voluntad soberana del pueblo", ente geométrico, pétreo y perfecto. Por ende, la Constitución como pináculo de la pirámide positivista es la representación y el mayor paradigma del derecho moderno.

Para los positivistas-legalistas del siglo XIX y de un bien avanzado siglo $\mathrm{XX}$, la Constitución fue un texto perfecto, cerrado, en el que no hay lugar para lagunas o antinomias. Siguiendo este hilo argumentativo, la Constitución moderna es concebida como el receptáculo de la voluntad popular soberana, como un fiel reflejo de la realidad social, cultural, política e, incluso, económica.

Por todo lo anterior, en la modernidad se entendió que las Constituciones son supremas, como reflejo de la soberanía y la voluntad popular, como fuente de legalidad y validez del resto del sistema normativo y como fuente de las competencias y límite al poder de la autoridad.

Sin embargo, como todos los paradigmas, llega un momento en que las ideas jurídicas de la modernidad son fuertemente cuestionadas.

Si en la modernidad, la ciencia prometió alcanzar el progreso, la verdad y la felicidad; en la postmodernidad estos ideales se desvanecen: el progreso se cuestiona cuando éste se da a costa de la dignidad humana, la verdad y la infalibilidad de los conocimientos científicos se relativizan y la felicidad se ve, ahora más que nunca, como una utopía.

El posmodernismo alcanza al derecho. Cabe en este momento preguntarnos si los conceptos jurídicos de la modernidad corresponden con las realida- 
des sociales, culturales y políticas contemporáneas. Las respuestas pueden dar lugar a debate. A continuación aportamos nuestra posición al respecto.

Si hay algo que caracteriza nuestros tiempos es la tendencia, irreversible, a la globalización.

Los Estados se unifican, primero con intenciones económicas, pero este ánimo unificador genera una constante transculturización, nos convertimos poco a poco en una aldea global. Asimismo, al verse diluido el paradigma de un Estado centralista, supremo, soberano; se fortalecen las individualidades al interior del mismo, los regionalismos, los nacionalismos étnicos, las autonomías culturales y religiosas, salen a la luz y reclaman su derecho al reconocimiento, respeto y salvaguarda de sus diferencias y buscan que su diversidad se integre al sistema normativo y político.

Las Constituciones dejan de ser la cúspide de los sistemas normativos: por encima de ellas se posicionan las normativas internacionales y los ordenamientos comunitarios.

Poco a poco, el concepto de soberanía pasa a ser sustituido por la idea de integración regional.

Las Constituciones deben flexibilizarse. Debe derrumbarse el paradigma de una Constitución perfecta, cerrada, inmutable y rígida, para sustituirse con el ideal de una Constitución falible, abierta, reformable y flexible.

En otro orden de ideas, pero estrechamente vinculado a nuestro argumento central, encontramos dos fenómenos socio-culturales que deben ser tomados en cuenta para abordar la idea de sistema constitucional abierto.

En la modernidad se abrazó con fuerza la idea de un Estado absoluto hegeliano, sin embargo, este estado resultó ser un postulado teórico perfecto, pero práctica y fácticamente imposible. En cualquier Estado conviven diversos grupos sociales, étnicos, religiosos y si bien, el sistema jurídico es diseñado por y para una mayoría, los derechos y las representaciones culturales y políticas de las minorías no deben ser soslayados. Por ello, es frecuente encontrar que dentro de un sistema constitucional conviven diversas expresiones jurídicas.

La globalización y la integración regional ponen, aún más, de manifiesto la necesidad de incorporar dentro de los sistemas constitucionales estas representaciones, así se habla de apertura constitucional hacia arriba - hacia elementos supranacionales o internacionales-y de apertura constitucional desde abajo — a elementos autóctonos o de los pueblos originarios-. 
Reconocemos que en este punto, abandonar el paradigma moderno de la Constitución nos lleva, ineludiblemente, a abrazar un nuevo paradigma: la Constitución posmoderna.

Y ¿qué se debe considerar como una Constitución posmoderna?

En primer lugar, la constitución posmoderna debe entenderse como un texto falible. Abandonando el paradigma moderno: no todo lo que está en la constitución debe ser aceptado como un dogma de perfección jurídica y legitimación material. Existe la posibilidad de que al interior del texto constitucional se encuentren normas "materialmente" inconstitucionales, normas contradictorias entre sí. El constitucionalismo de la postmodernidad comprende la existencia de valores y principios supraconstitucionales que deben orientar la resolución de antinomias y lagunas. El texto será siempre perfectible, mejorable, adaptable a los valores y principios que una sociedad, en un determinado momento, considere preponderantes. Esto nos lleva a la segunda característica: la apertura constitucional.

$\mathrm{Al}$ abandonar el paradigma del derecho como sistema completo y cerrado, aparece la opción postmoderna de entender al sistema jurídico - $-\mathrm{y}$ especialmente al sistema constitucional - como un sistema abierto. La apertura constitucional debe darse tanto a elementos extra-normativos - como el derecho internacional o la norma comunitaria- como a elementos metanormativos - como los principios y los valores que cada pueblo o sociedad abraza en cada época determinada-

En el constitucionalismo de la posmodernidad, es cada vez más frecuente, encontrar textos constitucionales que reconocen la apertura de su letra, por ejemplo el artículo 16.1 de la Constitución portuguesa, el artículo 20. de la Constitución italiana, el artículo 2o. de la Constitución brasileña y el artículo 3o. de la Constitución peruana, entre otros varios casos de constituciones con menos de 30 años de antigüedad. En estos textos citados, de manera taxativa y ejemplificativa, encontramos referencia al carácter abierto del texto constitucional, especialmente en materia de derechos fundamentales y pluriculturalidad; en resumen, la letra de las Constituciones citadas señala que serán derechos fundamentales integrados al sistema normativo en cuestión, además de los consagrados expresamente por la Constitución, aquellos que se incorporen en virtud de tratados internacionales o resoluciones judiciales y, en algunos casos, se señala la naturaleza pluricultural 
del sistema normativo. ${ }^{25}$ Esta apertura constitucional permite incorporar, no sólo al texto, sino al sistema constitucional propiamente dicho, elementos normativos, jurídicos y axiológicos que permitan adaptar el sistema a la dinámica realidad política, social, cultural e, incluso, económica. Y esto nos lleva a la tercera característica de la Constitución posmoderna: la flexibilidad y la reformabilidad.

El paradigma moderno de la Constitución completa, perfecta y cerrada, debe reformularse desde una perspectiva de flexibilidad y reformabilidad. Las constituciones de nuestro tiempo deben ser textos que puedan reformarse de manera adecuada para mantenerse "vivas" y en correspondencia con la realidad social, cultural y política. En América Latina, se tiene un justificado temor en materia de flexibilidad constitucional. La historia reciente, plagada de dictaduras y vaivenes políticos, nos lleva a desconfiar, de manera casi natural, de nuestros legisladores o autoridades; esto debe cambiar. Si bien, propugnamos por una flexibilidad en materia de reforma constitucional debemos comprender que la cuestión de la reforma no debe darse a la ligera, como bien señala Pedro de Vega, la reforma a la Constitución debe ser políticamente posible y jurídicamente necesaria. ${ }^{26}$

El paradigma del constitucionalismo postmoderno nos lleva a concebir la Constitución, no como un texto pétreo, cerrado, perfecto e infalible, sino como un sistema normativo abierto, flexible y en resumen: vivo. Se derrumba el tótem sagrado del texto constitucional, de la adoración a la letra de la Constitución, de la veneración del papel y la tinta y se erige el nuevo paradigma del constitucionalismo: una Constitución abierta, mutable y dinámica.

En este punto del trabajo, estamos conscientes que un argumento de esta naturaleza nos lleva a enfrentar la realidad de que una Constitución mutable, flexible, viva puede llevar a reconocer el sistema constitucional ya no sólo como un sistema dinámico, sino como un sistema caótico.

25 Por ejemplo la Constitución portuguesa señala en su texto: "La República portuguesa es un Estado democrático basado en la soberanía popular, en el respeto y la garantía de los derechos y libertades fundamentales y en el pluralismo de expresión y en la organización política democrática". El artículo 2o. de la Constitución italiana dice: "La República reconoce y garantiza los derechos inviolables del hombre, ora como individuo ora en el seno de las formaciones sociales donde aquél desarrolla su personalidad y exige el cumplimiento de los deberes inexcusables de solidaridad política, económica y social".

26 Véase Vega García, Pedro de, La reforma constitucional y la problemática del constituyente, Madrid, Tecnos, 1985. 


\section{REFLEXIONES FINALES}

En el lenguaje común el adjetivo caótico puede llevar implícito, la mayoría de las veces, un cariz peyorativo. Sin embargo, uno de los objetivos principales de este trabajo es aportar argumentos para cambiar esta impresión.

Un sistema caótico es sencillamente aquel en el que es posible que pequeñas alteraciones tengan enormes efectos y en el que aunque exista una tendencia hacia algún lugar (que en el caso del derecho sería un ideal), hacia una especie de atractor, el sistema nunca se posiciona en él sino que merodea, vaga a su alrededor, con el peligro adicional de que ante un aparente pequeño cambio, se aleje de manera impredecible de éste. Los sistemas caóticos son sistemas abiertos, en los que una variedad de condicionantes afectan su devenir previsto.

Siguiendo estos argumentos, la Constitución abierta no es solamente aquella que recibe en su seno elementos extra o metanormativos, sino aquella que se construye día a día con la interpretación del juez constitucional; esto implica una dinámica, pero no una dinámica lineal, previsible en todos los casos; implica una dinámica caótica, donde si bien no se encuentran elementos totalmente definidos si se han de identificar tendencias a corto plazo. La aceptación de un sistema como caótico nos obliga a desconfiar de las predicciones y tendencias de largo plazo y a asumir una actitud prudente y atenta.

El presente trabajo es un intento de reconocer la presencia del caos en los sistemas y específicamente en los sistemas sociales como el derecho. Como se ha delineado en el segundo apartado del mismo, los sistemas cerrados y perfectos, estrictamente lineales, solamente existen en el mundo de las ideas, en las teorías. En el mundo real lo que se observa son sistemas dinámicos, caóticos.

Pero el caos no significa desorden o anarquía total. Si bien, a una vista profana o superficial, podría parecer que los sistemas denominados caóticos, carecen de orden o congruencia, en el fondo esto no es así, no se trata de sistemas que no tengan guía alguna, puede tratarse de sistemas deterministas (es decir, gobernados por leyes precisas) pero dado que su comportamiento depende de muchas variables, no se puede predecir con precisión su devenir. A estos se les denomina sistemas complejos.

El sistema constitucional, como base del derecho, debe ser comprendido como un sistema no lineal, complejo, caótico, cuyos atractores pueden identificarse como aquellos valores implícitos en el propio sistema, valores que 
pueden ser de seguridad, de libertad, de solidaridad o de equidad, valores que constituyen un sistema no solamente de creación o de interpretación de las partes del sistema, sino de su adecuación al caso concreto, de su vigencia y de su permanencia.

Estos valores, como atractores de un sistema caótico, son lo que le dan sentido, coherencia y relativa estabilidad, pero sobre todo representan un horizonte, la mirada al bosque que permite no perderse en la maleza. Y, de acuerdo a lo anterior, serán los jueces constitucionales quienes tendrán en sus manos, identificar los valores-atractores de nuestro sistema constitucional caótico y serán quienes cumplan con la imprescindible función de dar vida a un texto que, per se, es un instrumento estático, frío y externo a la realidad jurídica, social y cultural.

Así, el juez constitucional será el artífice de la coherencia del sistema constitucional complejo, dinámico y caótico por su naturaleza, identificando los valores-atractores del propio sistema, y convirtiéndolo así en el mejor reflejo posible de la sociedad a la que rige y en el mejor instrumento para el desarrollo de esa sociedad.

\section{FUENTES CONSULTADAS}

ACKerman, Bruce, We the people. 1. Foundations, Cambridge, Harvard University Press, 1991.

AlaÉz CORRAL, Benito, Soberanía constitucional e integración europea, consultado en http://www.uniovi.es/constitucional/fundamentos/prime ro/pdf/alaez.pdf(23 de agosto de 2009).

BALAGUER, María Luisa, "La interpretación de las normas y la posibilidad de una sola respuesta correcta”, en Morodo, Raúl y VEGA, Pedro de (coords.), Estudios de teoría del estado y derecho constitucional en honor de Pablo Lucas Verdú, Madrid, Dykinson-UCM, 2000, t. I.

BartolomeI, María Luisa y CANÇADO TRIndAde, Antonio (comps.), "Diversidad en la conceptualización de los derechos humanos: universalismo y diversidad cultural en América Latina", Estudios básicos de derechos humanos, San José, Costa Rica, Instituto Interamericano de Derechos Humanos IIDH, 1999, t. IV.

BIRD, C., An Introduction to Political Philosophy, Cambridge University, Cambridge, 2006. 
BÖCKENFORDE, Erns W., Estudios sobre derechos fundamentales, Madrid, Trotta, 2001.

Bodine, Jean, Les Six Livres de la République, París, 1576.

CereiJido, M., "Del caos de los demonios al caos de los biólogos", Revista Universidad de México, núm. 540, enero de 1996.

Collins, H. M., Changing Order: Replication and Induction in Scientific Practice, Londres, Sage, 1985.

D'Abro, A., The Rise of the New Physics, Nueva York, Dover, 1951.

DAVID, René, Los grandes sistemas de derecho contemporáneo, 6a. ed., París, Dalloz, 1974.

DASTON, Lorraine y PARK, Katharine, Wonders and the Order of Nature, Nueva York, Zone Books, 1998.

Oтто, Ignacio de, Derecho constitucional. Sistema de fuentes, Barcelona, Ariel, 1987.

Vega García, Pedro de, La reforma constitucional y la problemática del constituyente, Madrid, Tecnos, 1985.

ECO, Umberto, Los límites de la interpretación, Barcelona, Lumen, 1993.

—, Interpretación y sobreinterpretación, Cambridge, Cambridge University Press, 1995.

ERWIN, Laszlo, La gran bifurcación, Barcelona, Gedisa, 1990.

Francois, Charles, El cerebro planetario, Cuadernos Gesi-AATGSC, núm.12.

GADAMER, Hans-Georg, Verdad y Método, Salamanca, Ediciones Sígueme, 1991.

GARCÍA DE ENTERRÍA, Eduardo, "La Constitución como norma jurídica", en GARCÍA DE ENTERríA, Eduardo y PREDIERI, A. (dirs), La Constitución Española de 1978, Madrid, 1980.

GEIGER, T., Die Rechtsnatur der Sanktion. Der Grundbegriff und die ihn legitimierenden Zwecke als Charakteristika der Rechtsnatur, Berlín, Duncker und Humblot, 2007.

GLeICK, J., Chaos: Making a New Science, Nueva York, Penguin Books, 1987.

Gomes Canotilho, J. J.,0 Dereito constitucional, Almedina, Coimbra, 1993.

, ¿Revisar la/o romper la Constitución dirigente? Defensa de un constitucionalismo moralmente reflexivo", REDC, núm. 43, 1995. 
GRUN, Ernesto, "El derecho postmoderno. Un sistema lejos del equilibrio", Revista Telemática de Filosofia del Derecho, Argentina, http:// www.filo sofiayderecho.com/rtfd/numerol/posmoderno.html, consultada el 10 de agosto de 2006.

GuERrA FILHO, W. Santiago, Ensayos de teoría constitucional, FortalezaCeara, 1989.

HÄBERLE, Peter, Teoría de la Constitución como ciencia de la cultura, Madrid, Tecnos, 2000.

HACKING, Ian, El surgimiento de la probabilidad, Barcelona, Gedisa, 1995.

- Representar e intervenir, México, Paidós-UNAM, 1996.

Hanson, N. R., "Observación”, Olivé León y Pérez,-Ranzaz, Ana Rosa, Filosofia de la ciencia, México, Siglo XXI-UNAM, 1989.

HAYEK, FRIEDRICH V., Derecho, legislación y libertad, vol. III: El orden político de una sociedad libre, trad. de Luis Reig Albiol, Madrid, Unión Editorial, 1982.

HEGEL, Georg Wilhelm Friedrich, Lecciones de filosofia de la historia, Madrid, Alianza Editorial, 1999.

HobBes, Thomas, El Leviathan, 23a. ed., México, Fondo de Cultura Económica, 1999.

KHUn, Thomas, La estructura de las revoluciones cientificas, México, Fondo de Cultura Económica, Serie Breviarios, núm. 213, 2006.

—_ La función del dogma en la investigación científica, Valencia, Teorema, 1979.

— L L L tensión esencial, México, Fondo de Cultura Económica, 1982.

Laudan, Larry, Beyond Positivism and Relativism. Theory, Method, and Evidence, Westview Press, Boulder, 1996.

Loewenstein, Teoría de la Constitución, Madrid, Alianza, 1997.

LuCAS VERDÚ, Pablo, La Constitución abierta y sus enemigos, Madrid, Alianza, 1999.

—_ Pablo, "Alabanza y menosprecio de la Constitución Inglesa", Revista de la Facultad de Derecho de la Universidad de Oviedo, España, núm. 28, 1956.

MANDOKI, Katya, Estética cotidiana y juegos de la cultura, México, Conaculta-FONCA, 2008.

Martínez, Sergio F., De los Efectos a las Causas, México, Paidós- 
UNAM, 1997.

MirAndA, Jorge, Manual de direito constitucional, t. IV, Direitos Fundamentais, 2a. ed., Coimbra, 1993.

REALE, Giovanni y ANTISERI, Darío, Historia del pensamiento filosófico y cientifico, Barcelona, Herder, 1995, 3 vols.

RicoeU, Paul, Teoría de la Interpretación, México, Siglo XXI, 1995.

POPPER, Karl, La sociedad abierta y sus enemigos, 4a. reimp., trad. de Eduardo Loedel, Buenos Aires, Editorial Paidós, 1991.

Prigogine, Ilya, ¿Tan sólo una ilusión?, Barcelona, Tusquets Editores, 1983.

RORTY, Richard; Philosophy as Cultural Politics, Cambridge University, Cambridge, 2007.

RoY, Arundhati, The God of Small Things, Nueva York, Random House, 1997.

Russo, Eduardo Ángel, Teoría general del derecho. En la modernidad y la postmodernidad, Buenos Aires, Abeledo Perrot, 1995.

THURENER, P. W., Die graduelle Konstitutionalisierung der Europäischen Union, Tübingen, Mohr Siebeck, 2006.

VAtтimo, Gianni; Nihilism and Emancipation. Ethics, Politics and Law, Nueva York, Columbia University, 2004.

VELASCO GóMEZ, Ambrosio (coord.), El concepto de heurística en las ciencias y las humanidades, México, Siglo XXI-UNAM, 2000.

Vna Leeuwen, Henry G., The Problem of Certainty in English Thought, 1630-1690, Martinus Nijhoff (International Archives of the History of Ideas núm.3), The Hague, 1970.

WeStFall, Richard S., The Construction of Modern Science, Cambridge, Cambridge University Press, 1998.

Fecha de recepción: 8 de enero de 2010 .

Fecha de dictamen: 18 de enero de 2012. 